\title{
Calibration of DEM parameters on shear test experiments using Kriging method
}

\author{
Bednarek Xavier ${ }^{1 \star}$, Martin Sylvain ${ }^{1}$, Ndiaye Abibatou ${ }^{2}$, Peres Véronique ${ }^{1}$, and Bonnefoy Olivier ${ }^{1}$ \\ ${ }^{1}$ Centre SPIN, LGF UMR CNRS 5307, École Nationale Supérieure des Mines de Saint-Étienne, 158 Cours Fauriel, F-42023 \\ Saint-Étienne Cedex 2, France \\ ${ }^{2}$ Areva-Melox, B.P. 93124, 30203 Bagnols sur Cèze Cedex, France
}

\begin{abstract}
Calibration of powder mixing simulation using Discrete-Element-Method is still an issue. Achieving good agreement with experimental results is difficult because time-efficient use of DEM involves strong assumptions. This work presents a methodology to calibrate DEM parameters using Efficient Global Optimization (EGO) algorithm based on Kriging interpolation method. Classical shear test experiments are used as calibration experiments. The calibration is made on two parameters - Young modulus and friction coefficient. The determination of the minimal number of grains that has to be used is a critical step. Simulations of a too small amount of grains would indeed not represent the realistic behavior of powder when using huge amout of grains will be strongly time consuming. The optimization goal is the minimization of the objective function which is the distance between simulated and measured behaviors. The EGO algorithm uses the maximization of the Expected Improvement criterion to find next point that has to be simulated. This stochastic criterion handles with the two interpolations made by the Kriging method : prediction of the objective function and estimation of the error made. It is thus able to quantify the improvement in the minimization that new simulations at specified DEM parameters would lead to.
\end{abstract}

\section{Introduction}

Mixing of powders is an important process unit in several industries e.g. pharmaceuytical, food and nuclear industry. The Discrete-Element-Method (DEM) [1] can simulate such processes and help their understanding and optimization. However the calibration of micromechanical parameters remains the most sensitive issue of the modeling process.

There is currently no clear link between micromecanical parameters used in DEM and macromechanical measured ones. It appears that mixing behaviors of different powders can be related to different behaviors in shear testers as the Freeman Technology FT4 rheometer. The choice of shear test as calibration experiment seems thus suitable. It appears that DEM simulations of shear testers are mainly influenced by the Young's modulus and the static friction coefficient [2-4].

The aim of this paper is to present a calibration methodology using Efficient Global Optimization (EGO) algorithm based on Kriging interpolation method. The methodology is applied on the couple of DEM parameters Young 's Modulus and static friction coefficient.

Particular importance is given in this work to determine the number of particles to be used in the DEM simulation of the shear tester.

\footnotetext{
^e-mail: xavier.bednarek@emse.fr
}

\section{Theoretical background \& methodology}

\subsection{Discrete element modeling method}

DEM describes the motion of spherical particles by integrating classical Newton's law in consecutive time steps. Positions, velocities and forces are updated at each time step. Contact-force models have been largely described in literature [5-7]. Here we use the most classical ones : the non-linear damped Hertz-Mindlin model for normal and tangential forces. Model of rolling friction [8] is used to represent the non-sphericity of particles. Viscous damping part of torque produced by rolling friction is negected. No cohesive forces are considered, but gravity is taken into account. Simulations were conducted with the open source software LIGGGHTS.

Choice of suitable time-step to integrate the motions is critical. The Rayleigh time step is often used when DEM simulations of dense regime are made. It represents the propagation time of a Rayleigh surface wave around a particle. As it is impossible in DEM paradigm to model such wave - the surface of an unique particle does not exist we consider here that the validity of such a time step is a questionable. We thus use the critical time step given in (1).

$$
\Delta t_{\text {crit }}=R\left(\frac{4}{3}\right)^{\frac{2}{3}} \pi^{\frac{1}{3}} \sqrt{\rho} \sigma_{0}^{-\frac{1}{6}} \tilde{E}^{-\frac{1}{3}}
$$

$\tilde{E}=E /\left(1-v^{2}\right)$ being the reduced Young's modulus. The other symbols meaning can be found in table 1. Calcula- 
tion of this time step is based on [9]. It represents the time needed for a perturbation of force propagating through a chain of identical particles in - Hertz - contact to cross a single one. It has the advantage of showing dependence on the compression $\sigma_{0}[\mathrm{~Pa}]$ of the chain. The resolution time step used here is about $5 \%$ of this critical time step.

As the size of the mixer - used in the target simulation - is fixed and as the computer/software performances limit the number of particles as about a million, the particle radius is fixed. This means that it is not conceivable to simulate one single real grain by one single numerical particle. Thus, the main challenge is to represent the rheology of a set of small grains with a set of larger numerical particles. It so is a critical point of first determine how many particles are needed to catch a powder behavior. Hence calibration experiments simulations are made with different number of particles, keeping constant the ratio between height and diameter of the shear cell and all DEM micromechanical parameters. From a certain number of particles, the result of the simulation no longer varies. The minimum number of particules to be used is therefore reached.

\subsection{The standard shear test using the FT4 Freeman Technology rheometer}

The standard shear cell technique for powder is based on the Jenike ring shear tester[10]. However unlike [3, 4], it is the cylindrical shape that is used here - as represented on figure 1 - particularly because the Freeman Technology FT4 rheometer is largely used in industrial application. Powder is placed into the shear cell and before shear a vertical stress $\sigma$ is applied by the shear head to pre-compress the material sample. The shear head rotate at constant velocity, maintaining normal stress constant, and the shear stress $\tau$ is measured. Four shear steps are made with four differents vertical stresses : successively $9 \mathrm{kPa}, 8 \mathrm{kPa}, 7$ $\mathrm{kPa}$ and $6 \mathrm{kPa}$. Before each shear step, a preshear step is made at $\sigma=15 \mathrm{kPa}$.

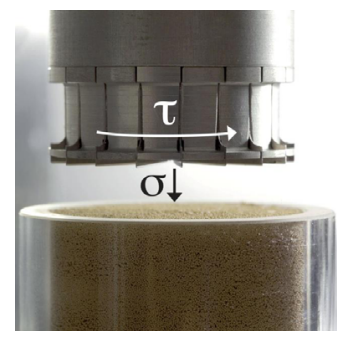

(a) FT4 rheometer

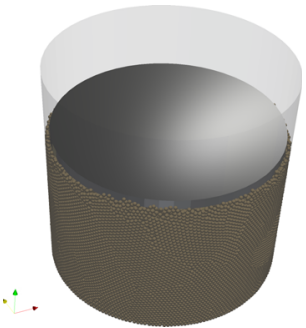

(b) DEM simulation
Figure 1. Shear tester used as calibration experiment

The effective measure is the yield locus of the powder, associated with the level of pre-compression. Indeed, each shear step should exhibit a clear maximum of measured shear stress before a strong collapse. This so called incipient faillure point corresponds to the critical point when the powder starts to flow under the shear stress applied. These four maximum shear stresses form the so called yield locus. Plot of these values versus the corresponding normal stress applied shows usually a linear trend. The slope is known as the angle of internal friction (AIF $\left[^{\circ}\right]$ ) and the Yintercept is reffered at cohesion $\mathrm{C}[\mathrm{Pa}]$. This last number is the extrapolated shear stress resitance that the powder should produce when sheared at zero normal stress. These two numbers describing the yield locus are then used to identify the appropriate couple of DEM parameters previously mentionned.

\subsection{Efficient Global Optimization using Kriging}

The goal of the calibration process is to find the couple of DEM parameters - Young's modulus and static coefficient of friction - that would minimize the distance between the simulated yield locus and the measured one. This distance can be viewed easily in the 2D-space $\mathrm{C}$ vs AIF as shown below on figure 4. As DEM simulations can be strongly time consuming, it is of great importance to have to do the least possible simulations to converge to the optimum.

The Efficient Global Optimization [11] is an optimization technique that is capable of choosing accuratelly new simulation points - new couples $\left(\mathrm{E}, \mu_{\mathrm{s}}\right)$ - to converge rapidly to the best solution. It can be viewed as a method to build progressively efficent design of experiments (DOE). The core of the method is to use Kriging interpolation [12] to predict the value of the objective function that has to be minimized - here the distance between simulated and experimental yield locus - and also the error made on this prediction. The smaller the prediction of this distance for a couple of DEM parameters and the the error on this prediction are, the bigger the chance of this couple to be choosen by the EGO algorithm to be the next point to be simulated is. In fact, it is the maximizatio of the Expected Improvment (EI) criterion (eq. 2) that allows the EGO algorithm to find this point.

$$
\begin{aligned}
\mathrm{EI}(\mathbf{x})=(\min (\mathbf{Y})- & \left.m_{\mathrm{K}}(\mathbf{x})\right) \Phi\left(\frac{\min (\mathbf{Y})-m_{\mathrm{K}}(\mathbf{x})}{s_{\mathrm{K}}(\mathbf{x})}\right) \\
+ & s_{\mathrm{K}}(\mathbf{x}) \phi\left(\frac{\min (\mathbf{Y})-m_{\mathrm{K}}(\mathbf{x})}{s_{\mathrm{K}}(\mathbf{x})}\right)
\end{aligned}
$$

In equation (2), $\mathbf{x}$ is the couple $\left(\mathrm{E}, \mu_{\mathrm{s}}\right)$ on which the $\mathrm{EI}$ is calculated, $\mathbf{Y}$ is the vector of all the distances between simulations and experiment that have already been calculated, $m_{\mathrm{K}}(\mathbf{x})$ and $s_{\mathrm{K}}(\mathbf{x})$ are respectivelly the prediction of the distance for the point $\mathbf{x}$ and the error on this prediction and $\Phi$ and $\phi$ are respectivelly the standard normal distribution function and its probability density function.

In fact, the EGO algorithm used here works on the minimization of the 2-points-EI criterion. This criterion is similar to the one presented above but it allows the selection of two new points to be calculated simulateously. It is thus possible to take advantage of using a cluster of computers to conduct the calibration/optimization. The algorithm used is implemented in the R-package DiceOptim [13]. 


\section{Results and discussion}

\subsection{How many grains in powder?}

Four simulations have been conducted with $12 \mathrm{k}, 31 \mathrm{k}$, $78 \mathrm{k}$ and $200 \mathrm{k}$ particles, that respectivelly correspond to 25 , 34, 46 and 63 particles per cell diameter. DEM parameter of these simuations are presented in table 1 . Correspond-

Table 1. DEM parameters values

\begin{tabular}{llll}
\hline Parameter & Symbol & Value & Unit \\
\hline Young's Modulus & $E$ & 25 & $\mathrm{GPa}$ \\
Static Friction Coef. & $\mu_{\mathrm{s}}$ & 0.5 & - \\
Poisson's Ratio & $v$ & 0.25 & - \\
Restitution Coef. & $e$ & 0.3 & - \\
Rolling Friction Coef. & $\mu_{\mathrm{R}}$ & 0.5 & - \\
Density & $\rho$ & 3000 & $\mathrm{~kg} \cdot \mathrm{m}^{-3}$ \\
Particle Radius & $R$ & 0.9 & $\mathrm{~mm}$ \\
\hline
\end{tabular}

ing yield loci shows on figure 2 a clear convergence. It appears that beyond $31 \mathrm{k}$ particles, the measured behavior does not change. This can be explained by the difference of compactness induced by wall effet. Compacity before shear steps are indeed larger for small number of particles per cell diameter. The nearer the walls are, the smaller the compacity is. And a compacter granular medium is more difficult to be sheared.

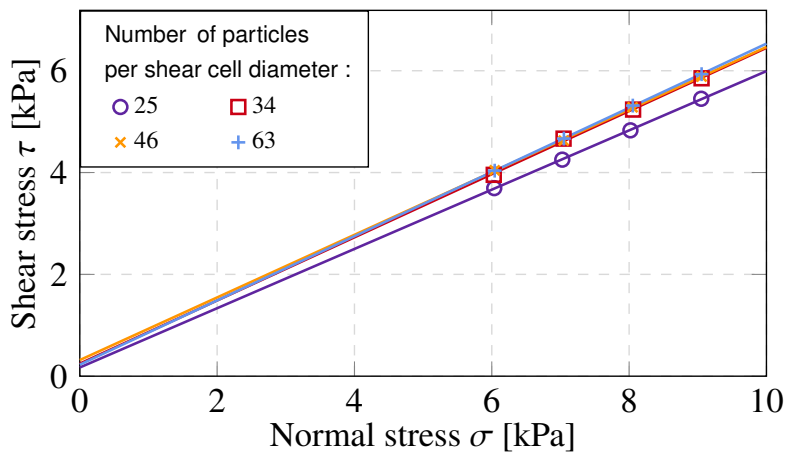

Figure 2. Yield loci of simulations with different numbers of particles

Figure 3 shows regular and smooth trends for the shear stresses measured. This is due to the non-friction wall hypothesis.

\subsection{Comparison with experimental measurements}

Given what has been observed above, calibration of DEM parameters has been started with $31 \mathrm{k}$ particles. Four simulations have been made to start the EGO algorithm. Figure 4 shows the result of these four simulations compared to experimental measurements made on two powders : $\mathrm{Al}_{2} \mathrm{O}_{3}$ with mean granulometry $88 \mu \mathrm{m}$ and $\mathrm{B}_{4} \mathrm{C}$ with mean granulometry $105 \mu \mathrm{m}$. Error bars for experiments are calculated from 3 repetitions of the full shear test and the ones for simulations are derived from classical statistics of linear regression.

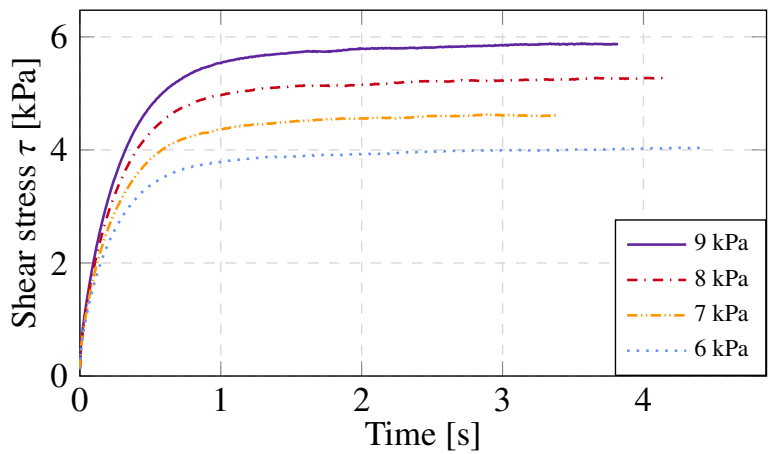

Figure 3. Shear steps for simulation with $78 \mathrm{k}$ particles

To find DEM parameters that lead to good calibration for $\mathrm{B}_{4} \mathrm{C}$, the two news points choosen by the EGO algorithm are $\left(E=33 \mathrm{GPa}, \mu_{\mathrm{s}}=0.29\right)$ and $\left(E=33 \mathrm{GPa}, \mu_{\mathrm{s}}=0.21\right)$. This choice can be easily comprehensible regarding figure $4: \mathrm{B}_{4} \mathrm{C}$ point is obviously above the iso-Young's modulus line at $25 \mathrm{GPa}$, and near the iso-static friction line 0.25 . As $\mathrm{B}_{4} \mathrm{C}$ is very close to this line, the algorithm tends to look around.

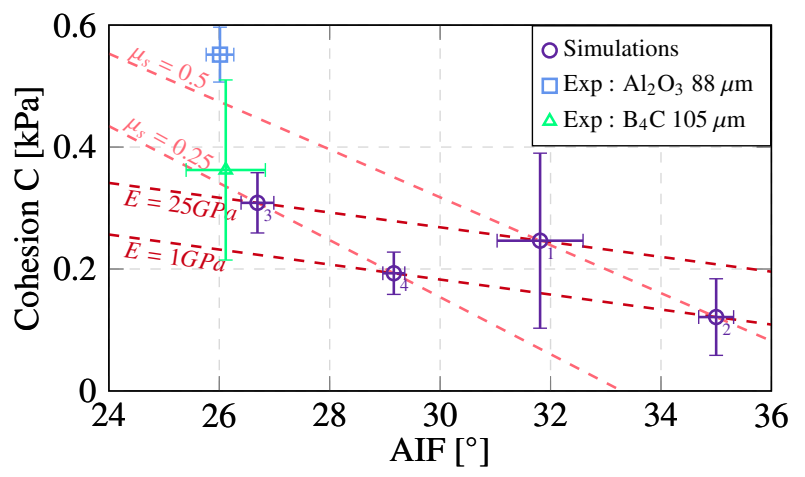

Figure 4. Cartography of the optimization

Figure 5 shows shear stress simulated at $9 \mathrm{kPa}$ normal stress for both these new points compared to experimental ones. However it should be noted that these curves are not the result of a calibration that would have converged. Order of magnitude are reached, but the shape of curves are not very similar. Experimental curves for $\mathrm{B}_{4} \mathrm{C}$ and $\mathrm{Al}_{2} \mathrm{O}_{3}$ show linear tends at the beginning, although simulations present much more concave shape. Experiment with glass beads shows also such a concave shape at the beginning. The concave shape can thus be explained by the mono-sized or quasi-mono-sized nature of the granulometry used. Indeed, the more extended granulometry of $\mathrm{B}_{4} \mathrm{C}$ and $\mathrm{Al}_{2} \mathrm{O}_{3}$ allows much more granular rearrangement, retarding the rise of the shear stress.

Beyond the considerations about the number of particles made previously, the particle size distribution (PSD) seems to be a critical point. Indeed, catching the so called powder behavior might also need to take into account more preciselly the PSD. It seems not sufficient to get enough particles, but it seems also important to reproduce the realistic partial coordination numbers of the packing 
- ie the number of contact between particles of different sizes. The shear test made for the glass beads - that show a narrow PSD - is closer to the numerical results presented here, probably because of this.

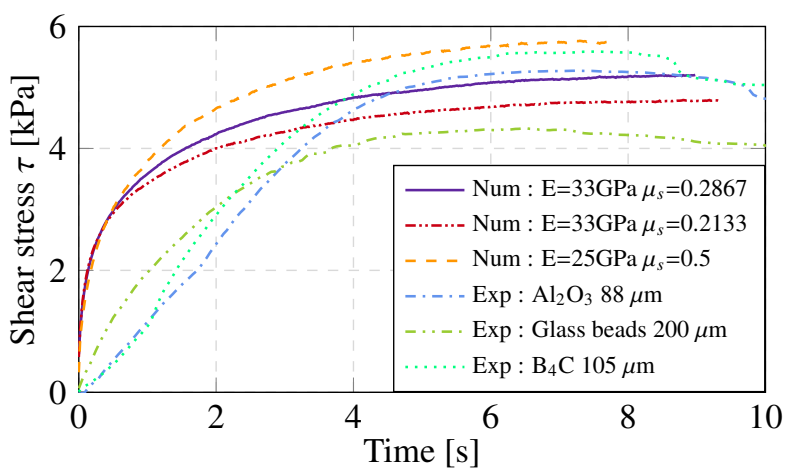

Figure 5. Comparison with experimental data at $\sigma=9 \mathrm{kPa}$

Moreover, the clear maximum of shear stress observed experimentaly is not reproduced by simulation. Here again this could be due to the mono-sized granulometry but recent simulations seems to show that there is a greater chance that this is due to the hypothesis of zero-friction at the walls of the shear cell. The work is currently moving in this direction.

\subsection{Conclusion}

Calibration of DEM parameters using the EGO algorithm seems promizing. The yield locus experimentally measured on powder sample can be approched by simulations. However, some assumptions made seem to be too restrictive to allow very good fitting of experimental curves : no friction at the wall and mono-sized granulometry. Future works must tackle these issues before continuing the proper use of the EGO algorithm.

\section{Acknowledgements}

This research was supported by Areva-Melox and Armines. This work was granted access to the HPC resources of IDRIS under the allocation 2016 - i2016097667 made by GENCI.

\section{References}

[1] P.A. Cundall, O.D. Strack, Geotechnique 29, 47 (1979)

[2] C. Coetzee, D. Els, Computers and electronics in agriculture 65, 198 (2009)

[3] T.A. Simons, R. Weiler, S. Strege, S. Bensmann, M. Schilling, A. Kwade, Procedia Engineering 102, 741 (2015)

[4] I. Keppler, F. Safranyik, I. Oldal, Engineering Computations 33, 742 (2016)

[5] J. Shäfer, S. Dippel, D. Wolf, Journal de physique I 6, 5 (1996)

[6] H. Zhu, Z. Zhou, R. Yang, A. Yu, Chemical Engineering Science 62, 3378 (2007)

[7] G.K. Barrios, R.M. de Carvalho, A. Kwade, L.M. Tavares, Powder Technology 248, 84 (2013)

[8] J. Ai, J.F. Chen, J.M. Rotter, J.Y. Ooi, Powder Technology 206, 269 (2011)

[9] C. Coste, E. Falcon, S. Fauve, Physical review E 56, 6104 (1997)

[10] A.W. Jenike, Bulletin of the University of Utah $\mathbf{5 3}$ (1964)

[11] D. Ginsbourger, Ph.D. thesis, École des Mines de Saint-Étienne (2009)

[12] G. Matheron, Les cahiers du centre de morphologie mathématique de fontainebleau (1969)

[13] O. Roustant, D. Ginsbourger, Y. Deville, Journal of Statistical Software 51, 54p (2012) 